\title{
Elastic response of the atomic nucleus in gauge space: Giant Pairing Vibrations
}

\author{
P.F. Bortignon ${ }^{1,2}$ and R.A. Broglia ${ }^{1,3, a}$ \\ 1 Department of Physics, University of Milan, Milan, Italy \\ 2 INFN Sez. di Milano, Milan, Italy \\ 3 The Niels Bohr Institute, University of Copenhagen, Copenhagen, Denmark
}

Received: 26 April 2016 / Revised: 6 July 2016

Published online: 14 September 2016

(c) The Author(s) 2016. This article is published with open access at Springerlink.com

Communicated by N. Alamanos

\begin{abstract}
Due to quantal fluctuations, the ground state of a closed shell system $A_{0}$ can become virtually excited in a state made out of the ground state of the neighbour nucleus $\left|g s\left(A_{0}+2\right)\right\rangle\left(\left|g s\left(A_{0}-2\right)\right\rangle\right)$ and of two uncorrelated holes (particles) below (above) the Fermi surface. These $J^{\pi}=0^{+}$pairing vibrational states have been extensively studied with two-nucleon transfer reactions. Away from closed shells, these modes eventually condense, leading to nuclear superfluidity and thus to pairing rotational bands with excitation energies much smaller than $\hbar \omega_{0}$, the energy separation between major shells. Pairing vibrations are the plastic response of the nucleus in gauge space, in a similar way in which low-lying quadrupole vibrations, i.e. surface vibrations with energies much smaller than $\hbar \omega_{0}$ whose eventual condensation leads to quadrupole deformed nuclei, provide an example of the plastic nuclear response in 3D space. While much is known, in particular concerning its damping, regarding the counterpart of quadrupole plastic modes, i.e. regarding the giant quadrupole resonances (GQR), $J^{\pi}=2^{+}$elastic response of the nucleus with energies of the order of $\hbar \omega_{0}$, little is known regarding this subject concerning pairing modes (giant pairing vibrations, GPV). Consequently, the recently reported observation of $L=0$ resonances, populated in the reactions ${ }^{12} \mathrm{C}\left({ }^{18} \mathrm{O},{ }^{16} \mathrm{O}\right){ }^{14} \mathrm{C}$ and ${ }^{13} \mathrm{C}\left({ }^{18} \mathrm{O},{ }^{16} \mathrm{O}\right){ }^{15} \mathrm{C}$ and lying at an excitation energy of the order of $\hbar \omega_{0}$, likely constitutes the starting point of a new field of research, that of the study of the elastic response of nuclei in gauge space. Not only that, but also the fact that the GPV have likely been serendipitously observed in these light nuclei when it has failed to show up in more propitious nuclei like $\mathrm{Pb}$, provides unexpected and fundamental insight into the relation existing between basic mechanisms - Landau, doorway, compound damping - through which giant resonances acquire a finite lifetime, let alone the radical difference regarding these phenomena displayed by correlated $(p h)$ and $(p p)$ modes.
\end{abstract}

\section{Introduction}

Systems displaying many degrees of freedom can be described at profit in terms of field theories of fermions and of bosons and of their interweaving [1,2]. Examples are provided by Quantum Electro Dynamics (QED) [3] and by Nuclear Field Theory (NFT) [4,5]. In QED, electrons and positrons are the fermions, photons are the bosons. In NFT, taylored after Feynman's version of QED in order to describe the nuclear structure in general, and that around closed shells in particular, the nucleons, namely particles $(p)$ and holes $(h)$ are the fermions, while correlated particle-hole $(p h),(p p)$ and $(h h)$ collective vibrations are the (composite) bosons.

\footnotetext{
a e-mail: broglia@mi.infn.it
}

In QED the photon field and the electron field are in interaction (fine structure constant). As a consequence, the identification of each field by these names is only an approximate one. What one calls physically an electron is only partially to be associated with the electron field alone. It is also partially to be associated with the photon field. Physically, an electron can sometimes radiate a photon and, at a later time, reabsorb it. Conversely, what one calls a photon, propagating through empty space can occasionally materialize itself in space and become replaced by an electron and a positron (particle $\left(e^{-}\right)$-hole $\left(e^{+}\right)$pair). Each of these fermions can radiate and reabsorb a photon (self-energy) or exchange it (vertex correction), and then, in the course of time, recombine to reform a photon. In other occasions, before the electron reabsorbs the radiated photon it can annihilate with the positron producing a sec- 
ond photon. These three-point vertex processes measure the scattering of light by light.

In NFT, the nucleon field and the vibrational fields are in interaction through the particle-vibration coupling vertices. A physical nucleon propagating in the nuclear medium can change orbital by bouncing inelastically off the nuclear surface and setting it into a $(p h)$ vibration, eventually reabsorbing it at a later time (self-energy process). Conversely, a correlated particle-hole can decay into one of its $(p h)$ components and eventually couple to $2 p$ $2 h$ states containing an uncorrelated $(p h)$ pair and a $(p h)$ collective vibration (doorway state). This vibration can either be reabsorbed by the same fermion which virtually excited it (self-energy) or be exchanged with the other fermion (vertex correction) before the particle $(p)$ falls into the hole $(h)$ and reconstitutes the collective vibration [6]. It can also propagate asymptotically and be joined by a second $(p h)$ collective vibration produced by the annihilation of the $p$ by the $h$, a process giving a measure of the interaction between one- and two-phonon vibrational states. It is at this point that the analogy between NFT and QED ends up.

In QED the entire effect of the scattering of light by an electric field is, to the lowest order, zero (Furry theorem). In a nutshell, any loop with an odd number of quanta in it is zero (ref. [7], p. 450). The above results are a consequence of the symmetry existing between particle (electron) and hole (positron) states.

If this were the case in nuclei, $(p, h)$ giant resonances, in general, and Giant Dipole Resonances (GDR), in particular, would display a damping width (lifetime) due solely to neutron- and $\gamma$-decay. Furthermore, multiphonon spectra would be harmonic. As a consequence, no $(p, h)$ vibrational state would display a finite value of the static quadrupole moment.

The above expectations are clearly contradicted by the experimental findings [8-10]. Giant resonances ( $p h$ elastic response of nuclei to impulsive fields) in general, and GDR in particular damp out, through coupling to doorway states, after few periods of oscillations, the associated width $\Gamma$ of few $\mathrm{MeV}$ being a direct measure of the asymmetry existing in nuclei between particle and hole states. In fact, if the damping mechanism was that resulting from $n$ - and $\gamma$-decay alone $\left(\Gamma_{n}^{\uparrow} / \Gamma \lesssim 10^{-1}, \Gamma_{\gamma_{0}} / \Gamma \lesssim 10^{-2},[11]\right)$, the damping width of a resonance would be a factor of at least 10 smaller than experimentally observed. Similarly, and again due to the asymmetry existing in nuclei between particle and hole states, low-lying $(p h)$ collective vibrations (plastic response of the nucleus to long lasting forces) in general, and $2^{+}$modes in particular, display sizable reorientation effects $([12])$, while multiphonon states show conspicuous anharmonicities [8]. This is in keeping with the fact that although the contributions arising from clockwise and anti clockwise three-point vertex processes describing the coupling between one- and twophonon states have opposite signs their summed value is finite $^{1}$.

\footnotetext{
1 Self-energy processes associated with the coupling of $(p h)$ giant resonances to doorway states containing e.g. a low-lying
}

Let us now turn to an example related to tunneling processes. In particular to the one-particle tunneling between a normal and a superconducting metal in weak contact, as compared to a $(d, p)$ reaction on a superfluid target nucleus. While the condensed matter expression of the associated current does not depend on the occupation factors $U_{k}^{2}$ ([14] and [15] p. 81), the nuclear one-particle transfer amplitude does [16]. This is in keeping with the fact in condensed matter, for a state $k$ above $\epsilon_{F}$ with energy $\epsilon_{k}$, there is a state $k^{\prime}$ below $\epsilon_{F}$ with $\epsilon_{k^{\prime}} \equiv-\epsilon_{k}$. Thus $U_{k}^{2}=V_{k^{\prime}}^{2}$ and $U_{k}^{2}+U_{k^{\prime}}^{2}=1$, a situation not encountered in the nucleus.

Summing up, in nuclei there is no symmetry between particle and hole states, as the last sentence of the abstract of [17] seems to imply and which seems to be at the basis of their research. Nonetheless, to learn about Giant Pairing Vibrations (GPV) $[17,18]$ is physically very important, and is comparable in relevance to that which is at the basis of studies of Giant Dipole (Pygmy) Resonances and low-energy $E 1$ modes in nuclei [19].

A large number of excited $0^{+}$states are known in the low-energy nuclear spectrum. Several mechanisms may produce collective states of this spin and parity. The best studied ones correspond to oscillations in the shape or in the size of the nucleus (so-called $(p h) \beta$ - vibrations in quadrupole deformed nuclei, and (two-quasiparticle) monopole states in superfluid spherical nuclei). These modes are associated with changes in the binding field of each particle, i.e. a field which conserves the number of particles. A special case of this type of modes is provided by the so-called coexistence states in $N=Z$ nuclei, in particular in ${ }^{16} \mathrm{O}$. The $0^{+}$state observed at $6.05 \mathrm{MeV}$ contains a large component of $4 p-4 h$ admixture of deformed configurations $[20,21]$.

In addition to the previous modes, nuclei display vibrations based on fields which create or annihilate two particles. Namely, vibrations in gauge space (pair addition and pair substraction modes) based on pairing fields associated with the pairing interaction and corresponding to two-particle $(p p)$ (two-hole $(h h)$ ) correlated modes. Because all of the associated configurations contribute in phase to the two-nucleon transfer form factors, these reactions are the specific tools to probe pairing vibrations. Suggested by Bohr in terms of the baryon (transfer) quantum number in early versions of $[8]^{2}$, see also [22], studied in terms of a simple model [23], implicitly included in spectroscopic studies of single-closed nuclei [24] and of $\beta$-vibrations in deformed nuclei [25], collective modes in

quadrupole collective vibration lead to contributions of the same sign both for the particle and the hole, and of the opposite sign for vertex correction contributions [13]. This is because particles and holes have opposite signs of the quadrupole moment, in keeping with the fact that closed shell systems, being spherical, have zero value for the quadrupole moment.

2 Within the context of the asymmetry between particles and holes states discussed above, it can be mentioned that the zero point amplitudes of the pair addition and pair removal modes differ from each other, since there is no symmetry connecting the two modes with transfer numbers \pm 2 (see [8], p. 392). 
gauge space were eventually formulated in detail in terms of pairing rotational and pairing vibrational bands [26] The predicted $4.95 \mathrm{MeV}$ two-phonon state of ${ }^{208} \mathrm{~Pb}$, product of the monopole pair removal and pair addition modes $\left(\left|{ }^{206} \mathrm{~Pb}(\mathrm{gs})\right\rangle \otimes\left|{ }^{210} \mathrm{~Pb}(\mathrm{gs})\right\rangle\right)$ was observed in the ${ }^{206} \mathrm{~Pb}(t, p)$ reaction, and the expected properties confirmed $([27,28]$, cf. also [29]). Within this context, it is to be noted that the low-lying $0^{+}$(coexistence) state of ${ }^{16} \mathrm{O}$ mentioned above is opposite to a multi-phonon pairing vibrational state, in keeping with the fact that deformation (low level density, Jahn-Teller-like phenomenon) opposes pairing (high level density phenomenon, [8], p. 386 and 641, [29]).

The low-lying collective pairing vibrations around closed shells, i.e. pairs of particles $(p p)$ and of holes $(h h)$, moving and correlating in the valence orbitals, have been studied in detail, and states made up to three pairing vibrational excitations have been observed [30,31]. These vibrations also dress the valence nucleons, mixing particle with hole states, and giving rise to retarded contributions to the state dependent effective mass [32-36], and to dealignments in deformed, rotating nuclei $[37,38]$.

Now, because of spatial quantization, single-particle levels in nuclei are bunched in major shells separated by an average energy $\hbar \omega_{0} \approx \frac{41}{A^{1 / 3}} \mathrm{MeV} \approx \frac{50 \mathrm{MeV} \mathrm{fm}}{R}$, where $R=1.2 A^{1 / 3} \mathrm{fm}$ is the nuclear radius. This is the origin of Giant Pairing Vibrations (GPV), that is (elastic) vibrations adding (removing) two nucleons and based on correlated $2 p(2 h)$ excitations across major shells ${ }^{3}$. One thus

${ }^{3}$ The frequency of elastic quadrupole vibrations of a solid sphere made out of particles of mass $m$ and density $\rho$ can be written as $\omega_{e l, Q}=(6 \mu / m \rho)^{1 / 2} /\left\langle r^{2}\right\rangle^{1 / 2}$, where $\mu$ is the Lamé shear modulus of elasticity, and $\left\langle r^{2}\right\rangle^{1 / 2}$ the mean square radius ([39], see also [40]). In nuclei, rigidity is provided by the energy difference $\hbar \omega_{0}\left(\approx 41 \mathrm{MeV} / A^{1 / 3}\right)$ between major shells. Within this context, the centroid of the giant quadrupole resonance can be written as $\hbar \omega_{G Q R}=\sqrt{2} \hbar \omega_{0} \approx 54 \mathrm{MeV} /\left\langle r^{2}\right\rangle_{\mathrm{fm}}^{1 / 2}(\approx$ $\left.58 \mathrm{MeV} / A^{1 / 3}\right)[10]$, similar in structure to the corresponding expression of $\hbar \omega_{G P V}[18]$, where the $A$-dependence testifies to the inverse dependence with the nuclear radius.

Both the low-lying quadrupole $(p h)$ and pairing $((p p),(h h))$ vibrations are mainly built out of $\Delta N=0$ jumps, $N$ indicating the major shell principal quantum number. Because the associated energies are rather small as compared to $\hbar \omega_{0}$, let alone $\epsilon_{F}$, it is not possible to write down an analytic expression which quantitatively reproduces the data as in the case of giant resonances, and one needs to make use of detailed microscopic (RPA, QRPA) calculations. Moving away from closed shell in both $N$ and $Z$, the energy of quadrupole vibrations decreases, and eventually no solution with positive energy is found (quadrupole deformation, phase transition, plastic behaviour). Coulomb excitation of a number of quadrupole phonons may eventually lead to fission [41,42].

Concerning the case of (low-lying) pairing vibrations, let us consider the modes based on ${ }^{208} \mathrm{~Pb}$. While the single pair removal mode $\left(\left|g s\left({ }^{206} \mathrm{~Pb}\right)\right\rangle\right)$ is well described in terms of RPA, the three-phonon state corrected by Pauli principle violation terms, and properly normalized, essentially coincides with the $n_{h}=3$ projection of the $\left|B C S\left({ }^{202} \mathrm{~Pb}\right)\right\rangle$ state $\left(n_{h}\right.$ indicating the number of hole pairs), thus providing an accurate description of deformation (plasticity) in gauge space. Within this context expects these vibrations to be found in all nuclei, disregarding whether they are normal or superfluid, spherical or deformed. GPV are expected to lie at an excitation energy of $\hbar \omega_{G P V} \approx 1.7 \hbar \omega_{0}$, and to carry a two-nucleon transfer cross section of the order of that associated with the low-lying (plastic) pairing vibrations. Predicted almost four decades ago ${ }^{4}$ [18], serious experimental candidates to the role of pair addition GPV have been found in a recently reported experiment ([17]; within this context, see also [44-46]), as the result of an experimental tour de force, backed by a systematic and less than straightforward theoretical calculation of the background. The importance of this work is that it ushers the experimental probing of the elastic response of the atomic nucleus in gauge space to state-of-the-art level, providing information about the associated elastic modulus, as well as concerning the effective two-nucleon transfer amplitudes (cf. fig. 1 of [18]), which parallel the nucleon effective charges, associated with $(p h)$ giant resonances, in particular with the $\mathrm{GDR}^{5}$ (cf. e.g. p. 486 of ref. [8]).

Before proceeding further let us make an assessment of the evidence for GPV presented by [17]. They have a sensible point on the oscillation of the angular distributions associated with the peaks at $13.7 \pm 0.1 \mathrm{MeV}\left({ }^{15} \mathrm{C}\right)$ and $16.9 \pm 0.1 \mathrm{MeV}\left({ }^{14} \mathrm{C}\right)$. While heavy-ion reactions are, as a rule, not the best probes to observe quantal effects, light heavy ions at the selected energy $(E=84 \mathrm{MeV})$ allow for a healthy interference between the distorted waves and an $L=0$ angular-momentum transfer pattern emerges. This is a fingerprint of the monopole GPV. An insight further corroborated by the absolute cross section of the resonance as compared with that associated with the ground state. For example, in the case of ${ }^{14} \mathrm{C}, \sigma(\mathrm{GPV}) \approx 0.66 \mathrm{mb}$ while $\sigma(g s) \approx 0.92 \mathrm{mb}$, again consistent with GPV although on the low side.

There are at least two objections one can level against the above arguments. The first concerns the fact that the observed resonance may correspond to a monopole $(p h)$ like excitation. However, arguably, only that of a correlated $(p h)$ excitation can carry a sizable two-nucleon transfer cross section of the order of that observed. But the monopole giant resonance (breathing mode) is expected at

we refer to the large anharmonicities found in the analysis of the multiphonon pairing vibrational spectrum in [43].

${ }^{4}$ Pairing vibrations are expected to be observed as distinct modes excited in two-nucleon transfer reactions with probabilities similar to those associated with single-particle transfer reactions exciting single-particle states, each time one can distiguish between particle and hole states, namely in the case of normal systems $\left(\left\langle g s\left|P^{\dagger}\right| g s\right\rangle=0\right.$, i.e. no static deformation in gauge space). This was the physical argument at the basis of the prediction of a universal giant pairing vibrational mode expected in all nuclei, in keeping with the fact that $\Delta \ll \hbar \omega_{0}$. This argument should not be confused with any symmetry between particle and hole states. Furthermore, the above physical argument makes it clear that nothing is gained (in principle only losed) in studying superfluid nuclei in the search for GPV.

5 The possibility to carry out similar studies is denied to Cooper pair transfer between metallic superconductors (no major shells; [47]). 
higher energies than observed [48]. Inelastic scattering (see e.g. [10]), in particular inelastic electron scattering [49] could provide important information on the above issue, in particular concerning the volume-surface structure of the form factor, to help clarify the question.

The second objection is likely more serious and reads somewhat as follows: why is that one can observe such an elusive mode as the GPV in light systems as ${ }^{14} \mathrm{C}$ and ${ }^{15} \mathrm{C}$ and not in heavy systems, where the phase space for the correlation of the two nucleons is much larger than in light nuclei, and where extensive search has been carried out without success. It is within this context that the results of [17] get further strength by connecting, unexpectedly, with a number of fundamental issues within the field of nuclear many-body physics.

Because of spatial quantization, shell effects are more important in light than in heavy nuclei. This is the reason why giant resonances suffer much stronger Landau damping $^{6}$ (breaking of strength) in the first than in the second type of systems (see e.g. [10], fig. 3.6).

On the other hand, actual damping (finite lifetime) is due to the coupling to doorway states and eventually to compound states ${ }^{7}$. And in this case the density of doorway states is much higher in heavy than in light nuclei ${ }^{8}$. Now, in the case of correlated $(p p)$ or $(h h)$ modes like the GPV, the damping widths of the two fermions add up instead of substracting as it happens in the case of correlated $(p h)$ giant pairing modes. Thus, GPV in heavy systems may acquire a very large width, incompatible with their detection as well defined states.

In the case of lighter systems, in particular of ${ }^{14} \mathrm{C}$, one may hypothesize that the $L=0$ resonance at $16.9 \mathrm{MeV}$ is a low-lying fragment of the GPV (after Landau damping), carrying a non-negligible fraction of the total two-nucleon transfer strength and having undergone a modest amount of doorway damping.

Recently, the question of the population of the GPV has been addressed [50]. The main conclusion of the paper is that "hot", in the two-nucleon transfer sense, configu-

\footnotetext{
${ }^{6}$ Landau damping in nuclei leads only to a dephasing of the different states in which the collective mode breaks at the level of (time-dependent) mean field.

7 The corresponding mechanism is illustrated in fig. 4.18 of [10]. Within the present context, it applies to each of the states in which the giant resonance breaks in due to Landau damping, i.e. to the splitting of the collective mode due to accidental degeneracies with unperturbed $(p h)$ roots (excitations).

8 For example, in the case of the GQR this density of $2 p-2 h$ states (containing an uncorrelated $p h$ excitation and a collective low-lying vibration all coupled to the right angular momentum and parity, i.e. $2^{+}$in the present case) is $\approx 20 \mathrm{MeV}^{-1}$ (see [10] pp. 87, 88 in particular fig. 4.16). The corresponding one in ${ }^{14} \mathrm{C}$ is almost an order of magnitude smaller. Concerning the density of $\mathrm{CN}$ states, the end point in the damping process, the difference amounts to $\rho\left(A=208, E^{*}=10 \mathrm{MeV}\right) / \rho(A=$ $\left.14, E^{*}=10 \mathrm{MeV}\right) \approx \exp [2(\sqrt{208}-\sqrt{14})] \approx 2 \times 10^{9}($ see $[10]$ p. 109). Important as these effects are, they can be overwhelmed by the fact that $V_{a \alpha}^{2}=V_{p}^{2}+V_{n}^{2}+V_{p} V_{n} \times$ (recoupling), $V_{a \alpha}$ indicating the coupling between the collective state $|a\rangle$ and the doorway state $|\alpha\rangle$ (see [10]).
}

rations namely $s_{1 / 2}^{2}(0)$ (also $p_{1 / 2}^{2}$ and eventually $p_{3 / 2}^{2}$ in the case of ${ }^{14} \mathrm{C}$ ) at threshold provide the largest effect which may cause dilution of the GPV strength, and not coupling to the continuum. This result is important for at least two reasons. The first, because it is consistent with experiments. This is in keeping with the fact that $(p h)$ giant resonances, which will be similarly affected by continuum effects as GPV, have been systematically observed throughout the mass table. The second reason is that the low- $l$ orbital effect is very similar to the so-called pairing anti-halo effect [51]. In the formulation of $[52,53]$ it takes place when $s, p$ states at threshold become unavailable for the HFB mean field. Because nuclei fulfilling such condition exist, e.g. ${ }^{11} \mathrm{Li}$, although with a ms lifetime, a longrange, eventually bootstrap-generated, pairing interaction is needed to stabilize the system [54-56]. Namely that arising from the exchange of the low-energy dipole mode and resulting in the binding, by $380 \mathrm{keV}$, of the two halo neutrons. In the process, a new elementary mode of excitation has been found: the (neutron) pair addition mode, which can be used as a building block in the construction of the nuclear spectrum. For example, as pairing excitation of the ground state of ${ }^{10} \mathrm{Be}$. In other words, arguably, the first excited $0^{+}$halo state $\left(E_{x}=2.25 \mathrm{MeV}\right)$ of ${ }^{12} \mathrm{Be}$ can be viewed as the $\left|g s\left({ }^{11} \mathrm{Li}\right)\right\rangle$ in a new environment (see [57], fig. A5).

It is possible that the reason why a pairing mode has been observed at $\approx 15 \mathrm{MeV}$ of excitation energy in $\mathrm{C}$ isotopes is that these modes are actually GPV (or Landau chunks of it) which, based mainly on the $s_{1 / 2}^{2}(0)$ and $p_{1 / 2}^{2}(0)$ configurations $^{9}$, have somewhat become neutron halo pair addition modes and, thus, a much more extended and diffused two-neutron-correlated configuration than that associated with the corresponding ground state. As a consequence, these extended states are expected to be resilient to coupling to other states and thus to damping or dilution. A tantalising question.

How to proceed? Likely, inelastic scattering $\left({ }^{14} \mathrm{C}\left(x, x^{\prime}\right)\right.$ $\left.{ }^{14} \mathrm{C}^{*}\right)$, one-particle transfer $\left(e . g .{ }^{13} \mathrm{C}(d, p){ }^{14} \mathrm{C}\right)$ as well as two-particle transfer $\left(e . g .{ }^{12} \mathrm{C}(t, p){ }^{14} \mathrm{C}\right)$. In this way one may be able to learn whether there are particularly collective $(p h)$ states on top of the $0^{+}$(dipole modes?), how important the $p_{1 / 2}^{2}(0)$ configuration in the GPV state is and eventually make clearer the $L=0$ oscillating pattern, respectively ${ }^{10}$.

The possibility to carry out systematic studies of GPV are expected to be instrumental in the test of two-nucleon transfer reaction mechanisms. Among other things, to get quantitative information concerning the relative role successive and simultaneous transfer play in the calculation of the absolute value of two-nucleon transfer differential cross

\footnotetext{
9 Within this context, it is noteworthy that the two neutron separation energies are $S_{2 n}=13.1 \mathrm{MeV}$ and $9.4 \mathrm{MeV}$ in ${ }^{14} \mathrm{C}$ and ${ }^{15} \mathrm{C}$ respectively.

${ }^{10}$ It does not escape our attention that the abundance of ${ }^{13} \mathrm{C}$ is only $1.07 \%$ and that tritium beams are past remembrance. On the other hand, inverse beam kinematics, gas targets and above all experimentalists' ingenuity may help.
} 
sections in a situation in which few single-particle $j^{2}(0)$ configurations contribute. Such information will in turn help shedding light on the spatial correlation of the nuclear Cooper pair partners [58-61], correlations which can also be estimated in terms of the Cooper pair quantality parameter $Q_{\text {pair }}$, generalization of the single-particle quantality parameter (see [57] and references therein). Namely, the ratio between the kinetic energy of localization within the correlation length $(\xi)$ and the correlation energy $E_{\text {corr }}$. That is $Q_{\text {pair }}=\frac{\hbar^{2}}{(2 m) \xi^{2}} \frac{1}{2 E_{\text {corr }}} \approx 0.03\left(\xi=\hbar v_{F} / 2 E_{\text {corr }} \approx\right.$ $\left.20 \mathrm{fm}, E_{\text {corr }} \approx 1.5 \mathrm{MeV}, v_{F} / c \approx 0.3\right)$. The above value implies a strong correlation between the Cooper pair partners. All these effects are rather subtle [14, 47, 62-66] and, at the same time, fundamental subjects needed to understand BCS superconductivity and superfluidity in fermionic systems at large and the GPV in particular.

The extension of monopole $\left(J^{\pi}=0^{+}\right)$GPV to other multipolarities and parities $\left(J^{\pi}=1^{-}, 2^{+}, 3^{-}, 4^{+}\right.$, etc. $)$ may likely be of importance in connection with the background and thus the intensity of the monopole GPV ${ }^{11}$ reported in [17], and will likely match that carried out in connection with the low-lying multipole pairing vibrations (cf. [66] p. 108). It is noteworthy that these (plastic) vibrations renormalized by the GPV may play a central role in double charge exchange reactions like ${ }^{40} \mathrm{Ca}\left({ }^{18} \mathrm{O},{ }^{18} \mathrm{Ne}\right){ }^{40} \mathrm{Ar}[68]$, which is of interest in the quest to determine the value of the matrix element involved in the neutrinoless double $\beta$-decay, an important test of the standard model [69-72].

The existence of major shells with alternating parity and separated by energies of the order of $\hbar \omega_{0}(8-10 \mathrm{MeV})$, is also at the basis of $(p h)$ giant resonances. In particular of the GDR, namely the sloshing back and forth of neutrons against protons in an antenna-like motion with which an atomic nucleus absorbs energy from a $\gamma$-beam. This nuclear excitation has been observed in essentially all nuclei throughout the mass table. Because one has to pay a conspicuous energetic price to separate protons from neutrons (symmetry potential), the energy centroid of the GDR lies at high energy in the nuclear spectrum, estimated to be $\hbar \omega_{D} \approx 2 \hbar \omega_{0}\left(\approx \frac{100 \mathrm{MeV} \mathrm{fm}}{R}\right)$. Its inverse proportionality to the nuclear radius testifies to the elastic character of the GDR.

It is interesting to note that one of today's growing points in nuclear research regards the study of low-energy $E_{1}$ strength found in nuclei with large neutron excess, that is, the study of the plastic response of the atomic nucleus to a dipole external field [19]. New and unexpected roles are found to be played by the associated low-energy fraction of the Thomas-Reiche-Kuhn sum rule [73,74] namely the giant dipole pygmy resonance (GDPR). A much stud-

\footnotetext{
11 It is noteworthy that in the present case, in which the doorway states leading to the breaking of the GPV strength consists in two uncorrelated particles and a collective low-lying vibration, both self-energy and exchange processes have the same sign [13]. Thus, the resulting width is expected to be much larger than that associated with e.g. the GDR. Within the context of the GPV background see also [67].
}

ied example is provided by the low-lying dipole mode of ${ }^{11} \mathrm{Li}([75]$ and references therein). This state acting as intermediate boson glues the halo neutron Cooper pair of ${ }^{11} \mathrm{Li}$ to the core ${ }^{9} \mathrm{Li}[54,56]$. It also provides new possibilities to test the Axel-Brink hypothesis. Hypothesis which posits that all nuclear states have a dipole mode on top of it $[76,77]$, and which plays an important role not only in the study of the nuclear structure, but also of the compound nucleus decay $[10,78]$. Last, but not least, the GDPR of ${ }^{11} \mathrm{Li}$ can be viewed as a correlated Cooper pair ${ }^{12}$ with quantum numbers $J^{\pi}=1^{-}$, arguably, the scenario of quantum vortex in nuclei ([79] and [66] appendix K; [80]). Insight into this question may be given with the help of the ${ }_{3}^{9} \mathrm{Li}_{6}(t, p){ }_{3}^{11} \mathrm{Li}_{8}\left(1^{-} ; 1 \mathrm{MeV}\right)$ two-nucleon transfer reaction, specific probe of pair addition modes in closed shell nuclei. Namely, in this case that associated with the magic number $N=6$, as a result of parity inversion.

Summing up, it may seem fair to state that the work of [17] has unlocked the doors of what, arguably, can become a precious laboratory to study nuclear many-body effects at large and pairing in particular, in a rather "clean" (few levels) light mass environment, similar to the one discovered by [55] in connection with two-nucleon transfer on ${ }^{11} \mathrm{Li}$ (see also [81]). Within this context, there exists a surprising and unexpected connection and physical unity of the studies of GPV (GDPR) lying at the forefront of today's nuclear research. That is, the mapping in gauge (isospin) space of the elastic (plastic) properties of this ever surprising drop of non Newtonian fluid, namely the atomic nucleus.

Discussions with Gregory Potel, Francisco Barranco, Enrico Vigezzi, Francesco Cappuzzello, Clementina Agodi, Manuela Cavallaro and Diana Carbone are gratefully acknowledged.

Open Access This is an open access article distributed under the terms of the Creative Commons Attribution License (http://creativecommons.org/licenses/by/4.0), which permits unrestricted use, distribution, and reproduction in any medium, provided the original work is properly cited.

\section{References}

1. S. Weinberg, The Quantum Theory of Fields, Vol. 1 (Cambridge University Press, Cambridge, 1996).

2. S. Weinberg, The Quantum Theory of Fields, Vol. 2 (Cambridge University Press, Cambridge, 1996).

3. R.P. Feynman, Quantum Electrodynamics (Benjamin, Reading, MA, 1962)

4. D.R. Bès et al., Phys. Lett. B 52, 253 (1974).

5. P.F. Bortignon et al., Phys. Rep. 30, 305 (1977).

6. G.F. Bertsch et al., Rev. Mod. Phys. 55, 287 (1983).

${ }^{12}$ In this discussion, the ground state and the GDPR of ${ }^{11} \mathrm{Li}$ is described as a $p_{3 / 2}(\pi)$ proton, playing the role of spectator, and a pair of correlated halo neutrons coupled to $J^{\pi}=0^{+}$and $J^{\pi}=1^{-}$, respectively. That is, $\left.\left.\right|^{11} \operatorname{Li}\left(g s ; 0_{\nu}^{+}\right)\right\rangle=\left|\tilde{0}_{\nu}\right\rangle \otimes\left|p_{3 / 2}(\pi)\right\rangle$ and $\left|{ }^{11} \operatorname{Li}\left(1^{-} ; 0.8 \mathrm{MeV}\right)\right\rangle=\left|1_{\nu}^{-}\right\rangle \otimes\left|p_{3 / 2}(\pi)\right\rangle[54]$. 
7. S.S. Schweber, QED (Princeton University Press, Princeton, New Jersey, 1994).

8. A. Bohr, B.R. Mottelson, Nuclear Structure, Vol. II (Benjamin, New York, 1975).

9. V.G. Soloviev, The Theory of Atomic Nuclei (Institute of Physics Publishing, Bristol, 1992).

10. P.F. Bortignon et al., Giant Resonances (Harwood Academic Publishers, Amsterdam, 1998).

11. T. Aumann et al., Annu. Rev. Nucl. Part. Sci. 48, 351 (1998).

12. J. de Boer, J. Eichler, Adv. Nucl. Phys. 1, 1 (1968).

13. P.F. Bortignon et al., Nucl. Phys. A 398, 221 (1983).

14. M.H. Cohen et al., Phys. Rev. Lett. 8, 316 (1962).

15. J.R. Schrieffer, Superconductivity (Benjamin, New York, 1964).

16. A. Idini et al., Phys. Rev. C 92, 031304 (2015).

17. F. Cappuzzello et al., Nat. Commun. 6, 6743 (2015).

18. R.A. Broglia, D.R. Bes, Phys. Lett. B 69, 129 (1977).

19. D. Savran et al., Prog. Part. Nucl. Phys. 70, 210 (2013).

20. G.E. Brown, in Comptes Rendus du Congrès International de Physique Nucléaire, Vol. 1 (Centre National de la Recherche Scientifique, Paris, 1964) p. 129.

21. T. Engeland, Nucl. Phys. 72, 68 (1965).

22. A. Bohr, Elementary modes of excitation and their coupling, in Comptes Rendus du Congrès International de Physique Nucléaire, Vol. 1 (Centre National de la Recherche Scientifique, Paris, 1964) p. 487.

23. J. Högaasen-Feldman, Nucl. Phys. 28, 258 (1961).

24. R. Arvieu et al., Phys. Lett. 4, 119 (1963).

25. D.R. Bès, Nucl. Phys. 49, 544 (1963).

26. D.R. Bès, R.A. Broglia, Nucl. Phys. 80, 289 (1966).

27. J.H. Bjerregaard et al., Nucl. Phys. 89, 337 (1966).

28. R.A. Broglia, C. Riedel, Nucl. Phys. 92, 145 (1967).

29. B.R. Mottelson, Elementary Modes of Excitation in $\mathrm{Nu}$ clei, Le Prix Nobel en 1975 (Imprimerie Royale Norstedts Tryckeri, Stockholm, 1976) p. 80.

30. E.R. Flynn et al., Phys. Lett. B 41, 397 (1972).

31. R.A. Broglia et al., Adv. Nucl. Phys. 6, 287 (1973) (www. mi.infn.it/ vigezzi/BHR/BrogliaHansenRiedel.pdf).

32. D.R. Bès, R.A. Broglia, Phys. Rev. C 3, 2349 (1971).

33. E.R. Flynn et al., Phys. Rev. C 3, 2371 (1971).

34. D.R. Bès, R.A. Broglia, Phys. Rev. C 3, 2389 (1971).

35. R.A. Broglia et al., Phys. Lett. B 50, 213 (1974).

36. R.P.J. Perazzo et al., Nucl. Phys. A 339, 23 (1980).

37. F. Barranco et al., Phys. Lett. B 198, 19 (1987).

38. Y.R. Shimizu et al., Rev. Mod. Phys. 61, 131 (1989).

39. G.F. Bertsch, R.A. Broglia, Oscillations in Finite Quantum Systems (Cambridge University Press, Cambridge, 2005).

40. G.F. Bertsch, Sci. Am. 248, 62 (1983).

41. Karin Beyer, Aa. Winther, Phys. Lett. B 30, 296 (1969).
42. Hans Kruse et al., Phys. Rev. C 22, 2465 (1980).

43. R.M. Clark et al., Phys. Rev. Lett. 96, 032501 (2006).

44. G.M. Crawley et al., Phys. Rev. Lett. 39, 1451 (1977).

45. G.M. Crawley et al., Phys. Rev. C 22, 316 (1980).

46. B. Mouginot et al., Phys. Rev. C 83, 037302 (2011).

47. B.D. Josephson, Phys. Lett. 1, 251 (1962).

48. D. Lebrun et al., Phys. Lett. B 97, 358 (1980).

49. M. Wakasugi et al., Nucl. Instrum. Methods Phys. Res. Sect. B 317, 668 (2013).

50. M. Laskin et al., Phys. Rev. C 93, 034321 (2016).

51. K. Bennaceur et al., Phys. Lett. B 496, 154 (2000).

52. Ikuko Hamamoto, Ben R. Mottelson, Phys. Rev. C 68, 034312 (2003).

53. I. Hamamoto, B.R. Mottelson, Phys. Rev. C 69, 064302 (2004).

54. F. Barranco et al., Eur. Phys. J. A 11, 385 (2001).

55. I. Tanihata et al., Phys. Rev. Lett. 100, 192502 (2008).

56. G. Potel et al., Phys. Rev. Lett. 105, 172502 (2010).

57. R.A. Broglia et al., Phys. Scr. 91, 063012 (2016).

58. M. Matsuo, Phys. Rev. C 73, 044309 (2006).

59. L. Ferreira et al., Nucl. Phys. A 426, 276 (1984).

60. M.W. Herzog et al., Phys. Rev. C 31, 259 (1985).

61. P. Lotti et al., Phys. Rev. C 40, 1791 (1989).

62. G. Potel et al., Rep. Prog. Phys. 76, 106301 (2013).

63. John Bardeen, Phys. Rev. Lett. 9, 147 (1962).

64. A.B. Pippard, The historical context of Josephson discovery. in 100 Years of Superconductivity, edited by H. Rogalla, P.H. Kes, (CRC Press, Taylor and Francis, Boca Raton, FL, 2012) p. 30.

65. P.W. Anderson, Special effects in suprconductivity, in The Many-Body Problem, edited by E.R. Caianello, Vol. 2 (Academic Press, New York, 1964) p. 113.

66. D. Brink, R.A. Broglia, Nuclear Superfluidity (Cambridge University Press, Cambridge, 2005).

67. P.F. Bortignon et al., Phys. Scr. 34, 678 (1986).

68. F. Cappuzzello et al., J. Phys.: Conf. Ser. 630, 012018 (2015).

69. J. Suhonen, O. Civitarese, Phys. Rep. 300, 123 (1998).

70. D. Frekers et al., Nucl. Phys. A 916, 219 (2013).

71. Vadim Rodin, Amand Faessler, Phys. Rev. C 80, 041302 (2009).

72. C.J. Guess et al., Phys. Rev. C 83, 064318 (2011).

73. F. Reiche, W. Thomas, Z. Phys. 34, 510 (1925).

74. W. Kuhn, Z. Phys. 33, 408 (1925).

75. R. Kanungo et al., Phys. Rev. Lett. 114, 192502 (2015).

76. D.M. Brink, PhD Thesis, Oxford University, 1955.

77. P. Axel et al., Phys. Rev. Lett. 19, 1343 (1967).

78. G.F. Bertsch, R.A. Broglia, Phys. Today 39, 44 (1986).

79. G.F. Bertsch et al., Nuovo Cimento 100, 283 (1988).

80. P. Avogadro et al., Phys. Rev. C 75, 012805 (2007).

81. I. Tanihata et al., Prog. Part. Nucl. Phys. 68, 215 (2013). 